Quaderni di Geografia Cahiers de Gécongaty rataphie

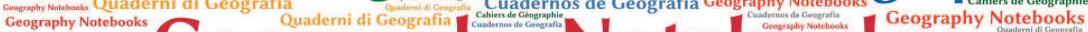
Cuadernos de Geografia Geography Notebooks $U 201000$ Cahiers de Géographi Cahiers de Géographie cahiers de Géographie Cuadernos de Geografía Cahiers de Géographie Cahiers de Géographie Cuadernos de Geografía Geography Notebooks

\author{
$4(2021)$ \\ 1 \\ Teatro di suoni. \\ Spazi acustici teatrali e territoriali
}

A cura di

Martino Mocchi, Lorena Rocca, Demis Quadri and Carlotta Sillano

EDITORIAL

Teatro di suoni per l'attaccamento ai luoghi. Uno sguardo geografico 11

Lorena Rocca

Per un teatro di suoni. Riflessioni su possibili dimensioni sonore nelle 23 creazioni site-specific di physical theatre

Demis Quadri

INTRODUCTION

Teatro di suoni. Spazi acustici teatrali e territoriali

Demis Quadri e Lorena Rocca

SPECIAL Issue

I suoni di Mantova come strumenti di interpretazione del paesaggio.

Tra turismo sostenibile ed educazione al patrimonio culturale

Valeria Pecorelli, Franca Zuccoli, Alessandra De Nicola, Enrico Squarcina

Il paesaggio sonoro campano tra contemporaneità e nuove forme

di progettualità turistica

Germana Citarella 
La narrazione spettacolarizzata del paesaggio sonoro.

Da Giuseppe Chiari a Philip K. Dick e oltre

Francesco Michi

Musica di paesaggi sonori. Enunciazione, risignificazione, comunicazione

Carlotta Sillano

Camminare per ascoltare. Partiture invisibili del territorio abitato

Elisabetta Senesi

Il paesaggio sonoro in relazione. Suono, movimento e immagini per stimolare complessità percettiva Angela Calia

Groove Fields. Understanding the Dance Floor from an Art-Based Research Perspective

Sebastian Mattbias

Il silenzio come esperienza trasformativa. L'importanza del silenzio nella meditazione e in ambito professionale

Sebastiano Caroni

Progettare il silenzio. Una lettura acustica dell'ex villaggio sanatoriale 125 Morelli a Sondalo

Martino Mocchi

Voicing One's Will. Theatre as Audio-Visual Hypotyposis of the Poetic

Michael Groneberg

Music and Clowning in Europe, 20th-21st centuries

Anna Stoll Knecht

Il paesaggio sonoro nella composizione musicale. Un percorso bibliografico

Stefano Alessandretti

\#exploreART: il labirinto di A. Pomodoro e i bambini. Un progetto di fruizione condivisa con percorsi sensoriali partecipati Alessandra De Nicola, Franca Zuccoli 


\section{OTHER EXPLORATIONS}

Il rumore lontano. Intervista a Lorena Rocca

a cura di Martino Mocchi

Re Cervo. Intervista a Antonella Astolfi

a cura di Krizia Bonaudo e Demis Quadri

Centovalli-Centoricordi. Intervista a Oliviero Giovannoni a cura di Krizia Bonaudo e Demis Quadri

Alla ricerca di un metodo: Open Space Technology 



\title{
Per un teatro di suoni. Riflessioni su possibili dimensioni sonore nelle creazioni site-specific di physical theatre
}

\author{
Demis Quadri \\ Accademia Teatro Dimitri SUPSI \\ DOI: https://doi.org/10.7358/gn-2021-001-quad
}

\begin{abstract}
Taking physical theatre as a reference point, in this paper I aim to explore the possibilities for a theatre that starts from sounds and places to develop its own forms, structures and contents. In the first part I explain what the expression "physical theatre" can mean, then I focus on the issue of space in theatre, before devoting a section to some peculiarities of site-specific approaches to the performing arts. The next step is to take into consideration certain aspects of the sound dimension in theatre, before outlining considerations that can help to start an exploration of a theatre of sounds that promotes new spaces for a real dialogue of this art with society.
\end{abstract}

Keywords: physical theatre; sounds; site-specific; places; spaces.

Parole chiave: physical theatre; suoni; site-specific; luoghi; spazi.

\section{INTRODUZIONE}

Prendendo come punto di riferimento il physical theatre, in queste pagine cercherò di esplorare le possibilità di un teatro che parta dai suoni e dai luoghi per sviluppare le proprie forme e i propri contenuti. L'ambizione è di abbozzare un'ipotesi che aiuti questo tipo di arte performativa a tro- 
vare nuovi spazi tra le forme espressive che dominano la nostra società, contribuendo al contempo - volendo essere molto ambiziosi - a sciogliere alcune fossilizzazioni che a partire dal Novecento hanno contribuito ad allontanare progressivamente il teatro dalla sfera delle forme di comunicazione artistica di maggiore impatto culturale (si pensi in particolare a quanto passa attraverso i supporti digitali e i social media).

Nella prima parte dell'articolo spenderò qualche parola per delineare cosa significhi l'espressione "physical theatre", che forse non è famigliare a tutti e che oltretutto ha in sé qualcosa di tautologico, essendo contraddittorio pensare a un teatro non fisico (Allain and Harvie 2014, 226). Successivamente mi soffermerò sul tema dello spazio a teatro, per fornire al lettore qualche chiave di lettura, prima di dedicare una sezione ad alcune peculiarità del site-specific. Il passo successivo sarà di prendere in considerazione certi aspetti della dimensione sonora nelle arti sceniche, prima di arrivare finalmente a considerazioni che possano avviare un percorso esplorativo verso un teatro di suoni che trovi nuovi spazi di dialogo con la contemporaneità.

\section{COS'È IL PHYSICAL THEATRE?}

Secondo lo storico Luigi Allegri, se pure nel teatro contemporaneo «lo spettacolo non è necessariamente la 'messa in scena' di un testo che gli preesiste, con questo orizzonte di attese dello spettatore che la cultura tradizionale del teatro ha depositato nelle nostre coscienze occorre comunque fare i conti in sede di analisi di uno spettacolo» (Allegri 2018, 18). Anche quando si parla di physical theatre è necessario tenere conto di questo medesimo orizzonte di attese, perché si fa riferimento a una forma di teatro che da un lato è lungi dall'essere universalmente nota al grande pubblico, dall'altro ha tra le proprie caratteristiche principali il fatto di partire, nei suoi processi compositivi, dal lavoro con e attraverso il corpo, anche quando si ispira a un testo drammatico (Callery 2001, 4).

A causa dei suoi modi di affrontare i processi di creazione, della sua storia e delle sue relazioni con prassi sceniche tradizionalmente separate (danza, arti circensi, performance art, ecc.), il physical theatre è difficile da definire in maniera chiusa. In un articolo su questo tema ho in effetti proposto un approccio aperto, che giocasse con le dinamiche e le tensioni tra un certo numero di dimensioni come testualità-corporalità, rappresentazione-performance, drammatico-postdrammatico, ecc. (Quadri 2020). Quello che importa qui è però che un elemento centrale per 
capire il physical theatre viene dall'intenderlo come un teatro dove il corpo e il movimento dell'attore sono al centro del processo drammaturgico: il corpo come strumento di lavoro, e il movimento come origine dell'amalgamarsi di forme e contenuti (Schmidt et al. 2018, 77). Ė proprio il fatto che il corpo dell'attore abbia una tale centralità nei processi di creazione e di composizione a rendere il physical theatre un terreno particolarmente fertile per sperimentare le potenzialità drammaturgiche della dimensione sonora in progetti site-specific. La centralità del corpo e del movimento in un processo di creazione artistica implicano il coinvolgimento delle relazioni profonde che queste componenti possono avere con spazio e suoni, due elementi che il privilegiare impulsi somatici più che costruzioni mentali (Callery 2001, 4) può aiutare a portare in primo piano, o a non relegare sullo sfondo, rispetto a impostazioni maggiormente legate a strutture narrative.

\section{LO SPAZIO A TEATRO}

Lo spazio è una componente di primaria importanza del fenomeno teatrale. Un regista fondamentale del teatro europeo, Peter Brook, intitola uno dei suoi libri più famosi Lo spazio vuoto e introduce la sua riflessione con queste parole: «posso scegliere uno spazio vuoto qualsiasi e decidere che è un palcoscenico spoglio. Un uomo lo attraversa e un altro osserva: è sufficiente a dare inizio a un'azione teatrale» (Brook 1998, 21). La coreografa, danzatrice e pedagoga Mary Overlie, ideatrice di un approccio alle arti performative noto come "Six Viewpoints", sviluppa un percorso che decostruisce il teatro (per poi ricostruirlo nel processo di creazione) in una serie di "punti di vista" - spazio, forma (shape), tempo, emozione, movimento e storia - che implicano nei loro rapporti reciproci una certa orizzontalità, ma che poi sembrano essere trattati in maniera piuttosto gerarchica nel fatto di intitolare il volume dove se ne parla Standing in Space (Overlie 2016).

Prima di concentrarmi più specificamente sul caso delle creazioni site-specific, è quindi utile che mi soffermi un momento su alcune prospettive che aiutano a leggere i tanto centrali aspetti spaziali del partecipare a un evento teatrale (come spettatori, attori o rappresentanti di altri mestieri della scena). Per farlo, seguirò e svilupperò alcune annotazioni di Paul Allain e Jen Harvie nell'articolo dedicato allo spazio del loro Routledge Companion to Theatre and Performance, al quale rimando per indicazioni bibliografiche sul tema (Allain and Harvie 2014, 248-250). 
Una classificazione piuttosto basica della questione può individuare tre aree fondamentali: lo spazio scenico (stage space), lo spazio del teatro (theatre space) e l'ambiente teatrale (theatre environment). Lo spazio scenico (o del palco), è riferito al luogo dove si svolge l'azione teatrale e alla sua scenografia. Nella sua relazione con la pratica nel teatro o nella danza, esso può essere interrogato chiedendosi ad esempio come faciliti o limiti i movimenti dei performer, o come influisca sulle possibilità di interazione tra questi ultimi. In termini semiotici può essere invece investigato prendendo in considerazione i suoi significati metaforici e finzionali, o riflettendo sul suo rappresentare spazi più o meno concreti e riconoscibili (una camera da letto, una foresta, una prigione, un castello, ecc.) o astratti (e capaci di un'evocazione meno diretta di oppressione, vitalità, romanticismo, desolazione o altro). Lo spazio del teatro è quello architettonico che comprende il palco e lo spazio del pubblico, e la prospettiva che lo considera permette di analizzare, per esempio, la relazione tra lo spettacolo e il pubblico tenendo conto della visuale, dell'acustica, delle posizioni reciproche dei diversi spettatori, degli arredamenti, dei servizi per il pubblico e per gli artisti, ecc. Questa dimensione può essere considerata anche nei suoi effetti in termini di inclusività e di accessibilità: il teatro dà una sensazione di accoglienza o di esclusione? Tratta gli spettatori di qualsiasi estrazione sociale e condizione economica allo stesso modo? È fruibile per persone con disabilità? Privilegia in qualche modo gli artisti di fama? E cosi via. L'ambiente teatrale (che cerca di tradurre l'inglese theatre environment) è uno spazio del teatro in senso più ampio, legato per esempio alla collocazione di una sede di spettacoli in una determinata posizione geografica: il teatro si trova in una metropoli, in un quartiere culturale, in periferia, in campagna, in un luogo di "pellegrinaggio" (come può essere Stratford-upon-Avon, città natale di William Shakespeare), vicino a un centro del potere politico, economico o religioso? Quale significato può avere questa sua collocazione?

\section{IL CASO SITE-SPECIFIC}

Spazio scenico, spazio del teatro e ambiente teatrale si intrecciano in modo particolare nel caso degli spettacoli site-specific. Questi si riferiscono, seguendo di nuovo Paul Allain e Jen Harvie, a performance prodotte in siti non teatrali e intese a relazionarsi in maniera diretta con il significato e la storia di quei luoghi, spesso con l'ambizione di rivolgersi anche a un pubblico non interessato all'arte teatrale (Allain and Harvie 
2014, 180). Il percorso attraverso il quale si può arrivare a coinvolgere spettatori che altrimenti non andrebbero a teatro passa proprio dall'esplicitare una focalizzazione molto chiara su un contesto che può essere considerato secondo l'idea di "luogo antropologico" nella definizione che ne dà l'antropologo Marc Augé, cioè di luogo identitario, relazionale e storico (Augé 2009, 60-62). Gli spettatori ai quali mi sto riferendo sono in effetti quelli che assistono a uno spettacolo site-specific non per particolare interesse artistico o estetico, ma per il legame che hanno con il luogo dove si svolge la performance e per il valore che questo ha nel loro darsi un'identità, nel loro relazionarsi con una determinata territorialità, nel loro affiliarsi a una certa narrazione storica.

In questo quadro, è interessante notare come spazio scenico, spazio del teatro e ambiente teatrale nelle produzioni site-specific interagiscono tra loro, creando legami inscindibili e imprescindibili nel proprio contribuire alle forme, alle strutture e ai contenuti dello spettacolo, ma anche come si intrecciano all'esperienza degli spettatori entrando in dialogo con i loro vissuti e modificandone la percezione del luogo stesso. «I luoghi - scrive Alice Giannitrapani - sono continuamente dinamizzati dagli individui che li percorrono e che, così facendo, gli conferiscono un senso e un valore. Di converso, attraversare gli spazi cambia i soggetti, ne ridefinisce lo statuto e, dunque, in parte, l'identità (2013, 34-35). Lo spettacolo site-specific si sviluppa dunque in una fitta rete dove più che altrove il mondo portato dalla messa in scena, quello portato dal pubblico e quello definito dal luogo si intrecciano in maniera drammaturgicamente rilevante, andando al di là delle normali condizioni per le quali sempre uno spettatore contribuisce al senso di un'opera d'arte e inevitabilmente uno spazio delimita le possibilità fisiche e simboliche di una rappresentazione teatrale. Si tratta insomma di un esempio privilegiato per esplorare come, prendendo le parole della grande teorica del teatro Erika Fischer-Lichte, «lo spazio performativo sia uno spazio permanentemente in divenire, nel quale la spazialità sorge attraverso le azioni e le percezioni di attori e spettatori» (Fischer-Lichte 2014, 201).

\section{LA DIMENSIONE SONORA}

La scelta del lavoro site-specific deve confrontarsi anche con la dimensione sonora, sia per quanto riguarda le sonorità che vengono dagli attori in scena (pianificate o meno, fatte di musiche, parole e rumori), sia per quelle prodotte dagli spettatori (consapevoli o meno, intenzionali o acci- 
dentali) o da altre persone o cose in movimento nell'ambiente circostante (e derivanti dal passaggio di mezzi di trasporto, dalle attività di un esercizio pubblico, dalle occupazioni degli animali in un parco, dall'evolversi di fenomeni atmosferici, ecc.).

Come ho già osservato altrove (Quadri 2017, 93), la dimensione sonora non è tra quelle che negli ambiti della prassi, della fruizione e della riflessione teatrali hanno goduto di un'attenzione esplicita straordinaria (salvo per certi aspetti nelle forme specificamente musicali di teatro), essendo in generale subordinata alla dimensione visiva. Ci sono naturalmente stati nel passato protagonisti della scena teatrale e artistica, come per esempio Antonin Artaud e László Moholy-Nagy, che hanno cercato di andare a fondo anche nell'approfondire la dimensione sonora della loro concezione di un teatro totale (Zavagna 2019, 45-48). E negli ultimi anni anche la ricerca accademica su questo aspetto del mondo delle arti sceniche sta trovando un suo spazio, dando origine a pubblicazioni come Noise, Water, Meat: A History of Sound in the Arts di Douglas Kahn (1999), Sound: A Reader in Theatre Practice di Ross Brown (2009), Theatre Noise: The Sound of Performance curato da Lynne Kendrick e David Roesner (2011), Drammaturgie sonore curato da Valentina Valentini (2012), Le son du théâtre curato da Jean-Marc Larrue e Marie-Madeleine Mervant-Roux (2016) e Teatri del suono a cura di Enrico Pitozzi (2018). Ma se pensiamo a un'idea stereotipata di teatro o se consideriamo l'insieme di quanto gli studi teatrali hanno prodotto nella storia, è probabile che il paesaggio sonoro non sia uno dei primi temi a emergere (cfr. Schafer 1977).

Erika Fischer-Lichte considera tuttavia la dimensione sonora come «paradigmatica della fugacità dello spettacolo teatrale. Cosa potrebbe infatti essere più fuggevole del (ri)echeggiare di un suono?» (Fischer-Lichte $2014,210)$. Per quanto la fugacità possa portare con sé i rischi di una certa fragilità, sonorità e teatralità possono però guadagnare da questo stesso aspetto in immediatezza e nel poter provocare, di conseguenza, effetti anche molto durevoli in chi li esperisce. Se le pratiche di physical theatre site-specific riescono a mettere a frutto queste potenzialità, possono sviluppare interazioni profonde con gli spazi che le ospitano e le sonorità che li vivono e che li definiscono nell'esperienza delle persone che li frequentano. Possono allora risultarne percezioni e prospettive diverse rispetto alle dinamiche e ai presunti confini di un luogo e dei suoi paesaggi sonori. In questo modo lo spazio sonoro che accoglie lo spettacolo teatrale, un po' come succede nello spazio estetico secondo Augusto Boal (Di Modica et al. 2005, 110-111), può svilupparsi in un'evoluzione 
all'insegna della varietà, della creatività e del cambiamento attraverso un intreccio con quanto vi proiettano attori e spettatori, dando la possibilità di sperimentare un luogo antropologico nel segno della dinamicità invece che di una staticità fatta di confini e di conservatorismo.

\section{Per Un TeAtro Di SUONI}

Le ultime considerazioni del paragrafo precedente non valgono soltanto per le potenzialità di un lavoro di physical theatre site-specific orientato su una interazione forte con il paesaggio sonoro che lo ospita. Tutto quello che fa parte di un luogo e che viene reinterpretato alla luce degli intrecci tra la sua storia, le sue caratteristiche, e le forme e i contenuti che vi vengono portati da uno spettacolo teatrale può conoscere gli stessi livelli di dinamicità e di creatività. Non va però trascurato il fatto che, come scrive Fischer-Lichte, il teatro "non è mai solo spazio della visione (theatron), $\mathrm{ma}$ anche spazio dell'ascolto (auditorium). In esso risuonano parole e canti, musica e rumori» $(2014,211)$. Se spesso, anche nel physical theatre e nel teatro di prosa (al di là della componente sonora delle parole), si è privilegiato il contributo dell'immagine alla costruzione di uno spettacolo, nulla vieta di rovesciare la logica mettendo prima la sfera dell'ascolto rispetto a quella della visione, un po' come avviene nell'apertura dello spettacolo di danza Cesena creato da Anne Teresa De Keersmaeker e Björn Schmelzer (2011), dove all'inizio lo spettatore nel buio percepisce soltanto i suoi prodotti dai performer nello spazio. Del resto un elemento fondamentale per qualsiasi evento scenico come il ritmo è stato frequentemente letto in termini di dinamiche tra suoni e immagini in pratiche e riflessioni che devono molto all'universo musicale (Ciancarelli 2006): si tratta di dinamiche che nei processi di creazione e di messa in scena hanno un ruolo strutturale, e il cui polo predominante può essere costituito tanto dalla dimensione sonora quanto da quella visiva.

Se pensiamo a un protagonista della storia delle arti sceniche che è considerato paradigmatico rispetto alla tradizione del teatro letterario, Konstantin Stanislavskij, possiamo notare come la sua ricerca sia fortemente incentrata sul principio di un lavoro dell'attore al servizio di personaggi e testi sviluppati da un autore (De Marinis 2013, 111-118). Nel caso del physical theatre ci troviamo invece di fronte a una metodologia di creazione scenica dove sono le esplorazioni dei performer a definire la costruzione di strutture, forme e contenuti teatrali (Quadri 2020). 
Purtroppo non c'è lo spazio in questa sede per presentare esempi concreti che illustrino un teatro che si costruisca a partire dai suoni, come potrebbero essere per certi aspetti i lavori della compagnia britannica Sound \& Fury o quelli del regista indiano B.V. Karanth (Sahai 2009), né per una descrizione verbale di come questo tipo di physical theatre site-specific possa funzionare (per esempio con attrici e attori che giocano con architetture e arredi urbani generando suoni con cui interagire, o con danzatrici e danzatori che si muovono sulla base di stimoli sonori dati dall'ambiente circostante). È ad ogni modo nel senso abbozzato nelle righe precedenti che credo si possa considerare il physical theatre come un ambito particolarmente promettente nella ricerca verso un teatro di suoni pensato in una logica site-specific. Il physical theatre può inoltre essere caratterizzato da un'inversione rispetto alla tradizionale gerarchia verticale che vede un chiaro percorso che dall'autore va verso il pubblico: nello specifico si parte invece da un'orizzontalità che permette varie possibilità di entrata nella creazione e nella sua strutturazione che portano, sulla base degli sviluppi del processo, a una verticalizzazione di contenuti, snodi drammaturgici e ruoli solo in un secondo tempo. Pensando alla varietà di materiali che possono fungere da punto di partenza per il physical theatre (Callery 2001, 5), tale orizzontalità rende quest'ultimo un candidato ideale per lo sviluppo di un teatro radicato nei suoni e nei luoghi delle performance. Il physical theatre si configura allora oltretutto come un'opportunità per mettere in discussione i preconcetti e gli stereotipi su come il teatro debba funzionare, aprendo le arti sceniche a nuove terre da esplorare per riaprire un dialogo profondo con la cultura e la società contemporanee attraverso una reinterpretazione dei luoghi, coi loro suoni, che queste stesse arti abitano.

\section{RIFERIMENTI BIBLIOGRAFICI}

Allain, P. and J. Harvie. 2014. The Routledge Companion to Theatre and Performance. London-New York: Routledge.

Allegri, L. 2018. Invito a teatro. Manuale minimo dello spettatore. Roma-Bari: Laterza.

Augé, M. 2009. Nonluoghi. Introduzione a una antropologia della surmodernità. Milano: Elèuthera.

Brook, P. 1998. Lo spazio vuoto. Roma: Bulzoni [trad. it. Isabella Imperiali].

Brown, R. 2009. Sound: A Reader in Theatre Practice. Basingstoke: Palgrave Macmillan.

Geography Notebooks - 4 (2021) 1

https://www.ledonline.it/Geography-Notebooks/ 
Callery, D. 2001. Through the Body. A Practical Guide to Physical Theatre. London: Nick Hern Books.

Ciancarelli, R., ed. 2006. Il ritmo come principio scenico. Ricerche e sperimentazioni del ritmo nel teatro e nella danza del Novecento. Roma: Dino Audino.

De Marinis, M. 2013. Il teatro dopo l'età d'oro. Roma: Bulzoni.

Di Modica, V., A. Di Rienzo e R. Mazzini. 2005. Le forme del gioco. Tecniche espressive per i laboratori interculturali. Roma: Carocci.

Fischer-Lichte, E. 2014. Estetica del performativo. Una teoria del teatro e dell'attore. Roma: Carocci.

Giannitrapani, A. 2013. Introduzione alla semiotica dello spazio. Roma: Carocci.

Kahn, D. 1999. Noise, Water, Meat: A History of Sound in the Arts. Cambridge: MIT.

Kendrick, L. and D. Roesner. 2011. Theatre Noise: The Sound of Performance. Newcastle: Cambridge University Scholars.

Larrue, J.M. and M.M. Mervant-Roux, eds. 2016. Le son du théâtre. XIXe-XXIe siècle. Histoire intermédiale d'un lieu d'écoute moderne. Paris: CNRS éditions.

Overlie, M. 2016. Standing in Space. The Six Viewpoints Theory \& Practice. Billings: Artcraft Printers.

Pitozzi, E., ed. 2018. Teatri del suono. Firenze: La Casa Usher.

Quadri, D. 2017. “Il paesaggio sonoro del teatro fisico”. Métis: bistória \& cultura 16 (32): 91-97. doi: 10.18226/22362762.v16.n.32.05

Quadri, D. 2020. "Le théâtre du corps. Pour une définition du terme physical theatre". In Philosopbies du jeu théâtral, edited by Michael Groneberg, 248-265. Lausanne: Unil.

Sahai, S. 2009. "Exploring a Theatre of Sounds". In Proceedings of Sound, Sight, Space and Play, edited by Wolf Motjie, 79-86. Leicester: De Montfort University.

Schafer, M.R. 1977. The Tuning of the World. Toward a Theory of Soundscape Design. Philadelphia: University of Pennsylvania Press.

Schmidt, Y., S. Marinucci, S. Bocchini, D. Quadri and A. Rey. 2018. "DisAbility on Stage - Exploring the Physical in Dance and Performer Training". Synnyt/Origins: Finnish Studies in Art Education. 2 (2018): 74-87. https://wiki.aalto.fi/ download/attachments/145101810/Yvonne_Schmidt_Sarah_Marinucci

Sara_Bocchini__Demis_Quadri_Anton\%20Rey.pdf?version=1\&modificationDate $=1550147900696 \&$ api $=\mathrm{v} 2$

Valentini, V., ed. 2012. Drammaturgie sonore. Teatri del secondo Novecento. Roma: Bulzoni.

Zavagna, P. 2019. "Paesaggio sonoro e composizione musicale: breve storia". In I suoni dei luoghi. Percorsi di geografie degli ascolti, a cura di Lorena Rocca, 37-78. Roma: Carocci. 